\title{
4D-STEM Differential Phase Contrast Microscopy Across Ferroelectric Domain Walls
}

Debangshu Mukherjee ${ }^{1}$, Colin Ophus ${ }^{2}$, Jim Ciston ${ }^{2}$, Zhiqiang $\mathrm{Mao}^{3}$, Venkatraman Gopalan ${ }^{1}$ and Nasim Alem $^{1}$

1. Department of Materials Science and Engineering, The Pennsylvania State University, University Park, PA.

2. National Center for Electron Microscopy, Molecular Foundry, Lawrence Berkeley National

Laboratory, Berkeley, CA.

3. Department of Physics and Engineering Physics, Tulane University, New Orleans, LA.

The dual wave-particle nature of electrons is the underlying idea behind Differential Phase Contrast (DPC) microscopy, where the wave nature of electrons is used to image and the charged particle nature of electrons is used to quantify electromagnetic potential gradients. In the past few years several breakthrough DPC experiments have been performed - which have demonstrated the potential gradient across single atomic columns with segmented detectors and the measurement of nuclear charge in GaN and $\mathrm{SrTiO}_{3}$ with 4D-STEM imaging [1,2]. However, both these experiments were performed with subangstrom aberration corrected electron probes, where the diffraction spots from the crystallographic axes overlap. While such a technique has the advantage of simultaneous atomic resolution imaging and DPC, as the 4D-STEM on $\mathrm{GaN}$ and $\mathrm{SrTiO}_{3}$ demonstrate - a noted shortcoming is the inability to measure long range electric fields arising from ionicity as the nuclear potential dominates the measurement. Additionally, the measurement accuracy for high resolution DPC is severely limited by sample thickness and works only when the sample is sub $5 \mathrm{~nm}$ thick [2].

The present investigation uses a large beam size, over a nanometer in diameter, to study the structure and charge accumulation across domain walls in the titanium doped hybrid improper ferroelectric $\mathrm{Ca}_{3} \mathrm{Ru}_{2} \mathrm{O}_{7}$ [3]. As demonstrated in Fig. 1a, the summed Bright Field STEM image from the underlying 4D-STEM data has some contrast variation at the domain wall, but the crystalline atomic structure is not visible anymore due to reduced resolution. Switching to smaller sub-angstrom beam size, we observe the presence of the $90^{\circ}$ domain walls (Fig. 1b) where the in-plane calcium displacements switch directions across the domain wall, with the polarization displacement shown by arrows overlaid on the STEM image. Since the entire collected data is a 4 dimensional data set $(2$ scanning dimensions in real space and 2 momentum dimensions in Fourier space), each and every scanning position is associated with a convergent beam electron diffraction (CBED) pattern as demonstrated in Fig. 1c. Plotting the CBED image with the intensities scaled in a logarithmic rather than a linear fashion demonstrates the presence of up to eighth order diffraction peaks, as shown in Fig. 1d. Since the rastered electron beam across the domain wall is larger than a nanometer, the diffraction spots do not overlap, allowing the measurement of the momentum change across the zeroth order diffraction disk with a sub-pixel accuracy in the Fourier dimension. The momentum variation across the $\mathrm{x}$ and $\mathrm{y}$ directions are shown for Figure 1e and $1 \mathrm{f}$ respectively which demonstrates the momentum reversal across a ferroelectric domain wall, indicating the presence of a localized variation in the projected potential right at the domain wall. This experiment demonstrates nanodiffraction DPC as an appropriate way to probe and further quantify the charge and potential variation across interfaces like ferroelectric domain walls with sub-nanometer precision [4].

References: 
[1] N. Shibata et al, Nature communications 8 (2017), p. 15631.

[2] K. Müller et al, Nature communications 5 (2014), p. 5653.

[3] N.A. Benedek and Turan Birol, Journal of Materials Chemistry C 4.18 (2016), p. 4000.

[4] D.M., V.G. and N.A. were funded by the Penn State Center for Nanoscale Science, an NSF MRSEC under the grant number DMR-1420620. Work at the Molecular Foundry was supported by the Office of Science, Office of Basic Energy Sciences, of the U.S. Department of Energy under Contract No. DEAC02-05CH11231.

a)

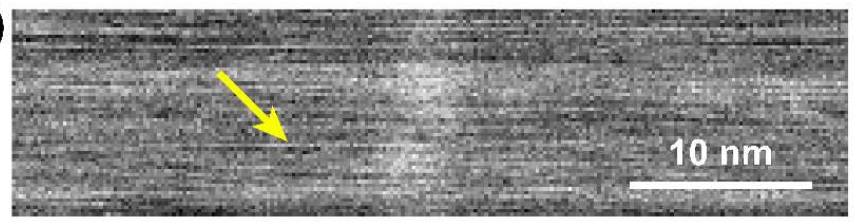

4D - STEM Calculated Bright Field

c)

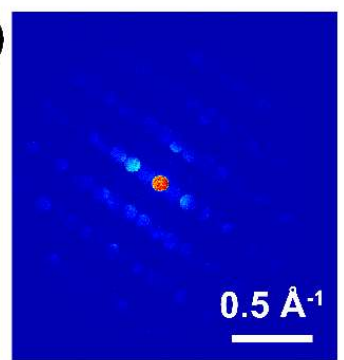

CBED from point d)

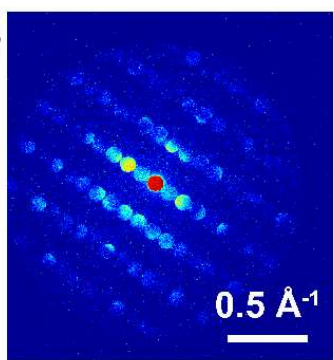

CBED (log scale intensity) b)

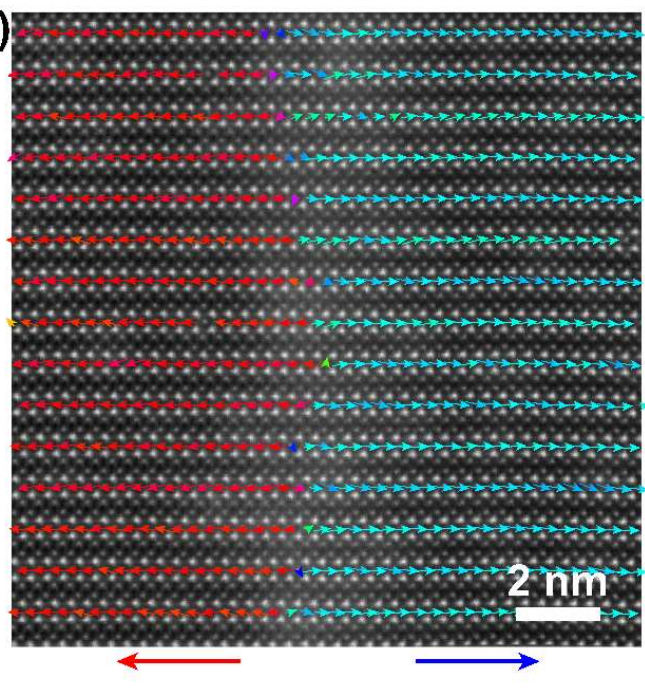

e)

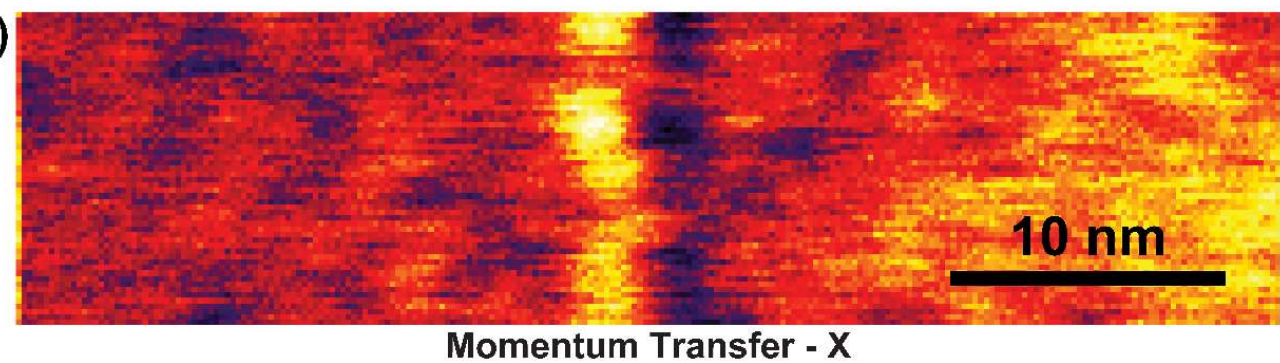

Momentum Transfer - X
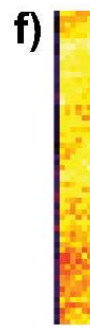

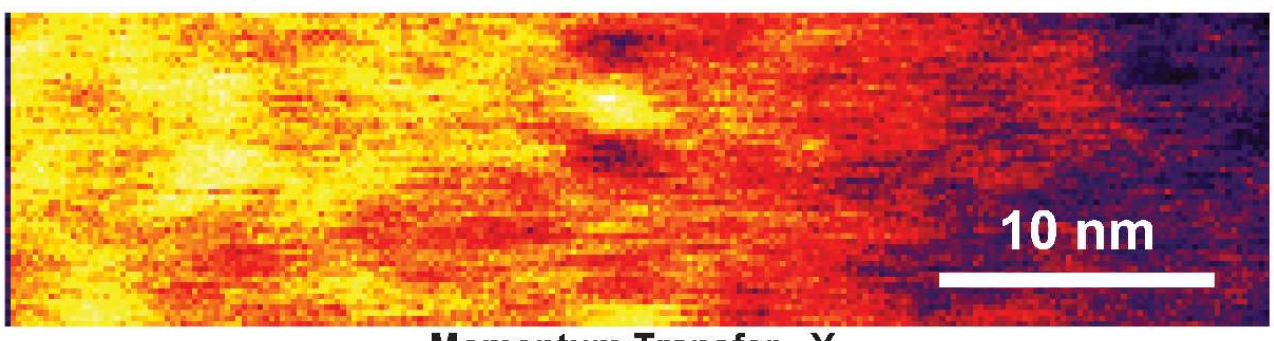

Momentum Transfer - $Y$
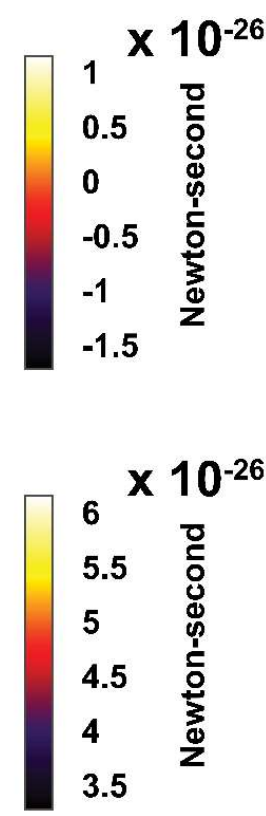

Figure 1. a) Calculated BF-STEM image from 4D-STEM dataset obtained by summing intensities up to $30 \mathrm{mrad}$. b) Aberration corrected ADF-STEM image with the polar displacement arrows overlaid. c) CBED pattern from the point referred to by the yellow arrow in Fig. 1a. d) CBED pattern in Fig. 1c with the intensity plotted logarithmically, demonstrating the existence of up to eighth order diffraction spots. e) Momentum transfer calculated from the central CBED disk along the $\mathrm{X}$ direction, i.e. perpendicular to the domain wall. f) Momentum transfer calculated from the central CBED disk along the Y direction, i.e. parallel to the domain wall. 